\title{
Una leche bubalina rica en ácidos grasos trans incrementó la colesterolemia y tendió a agravar la ateroesclerosis en conejos
}

\author{
Lertora, W.J. ${ }^{1}$; Villordo, G.I. ; Mussart, N.B. ${ }^{2}$; Catuogno, M.S. ${ }^{1}$; S.Negrette, M. ${ }^{1}$ \\ ${ }^{1}$ Cátedra de Patología General y Sistemática, ${ }^{2}$ Hospital de Clínicas, Facultad \\ de Ciencias Veterinarias, Universidad Nacional del Nordeste, \\ Sargento Cabral 2139, Corrientes (3400), Argentina. \\ E-mail: patgral@vet.unne.edu.ar
}

\begin{abstract}
Resumen
Lertora, W.J.; Villordo, G.I.; Mussart, N.B.; Catuogno, M.S.; S.Negrette, M.: Una leche bubalina rica en ácidos grasos trans incrementó la colesterolemia y tendió a agravar la ateroesclerosis en conejos. Rev. Vet. 30: 1, 32-38, 2019. Búfalas suplementadas con una mezcla de aceites de girasol + pescado pueden reducir en la leche los niveles de ácidos grasos saturados (AGS), considerados hipercolesterolémicos y pro-aterogénicos, e incrementar los ácidos grasos trans-11 18:1 y cis-9,trans-11 18:2, con propiedades anti-aterogénicas. Este trabajo comparó los efectos del consumo de dos leches bubalinas, con baja y alta relación de ácidos grasos trans (AGt)/AGS, sobre el lipidograma y el desarrollo de ateroesclerosis inducidos por colesterol en conejos. Veinte conejos neozelandeses, machos, fueron aleatoriamente separados en un grupo control $(\mathrm{n}=10)$ que recibió leche con baja relación AGt/AGS $(5,3 \%$ de AGt y $67,12 \%$ de AGS) obtenida de búfalas en sistema pastoril; y un grupo alto trans $(\mathrm{n}=10)$ que recibió leche con alta relación AGt/AGS (25,84\% de AGt y 45,89\% de AGS) obtenida de búfalas con suplementación lipídica en su dieta. La experiencia duró 75 días y, a partir del día 15 , todos los conejos fueron desafiados con $0,93 \mathrm{~g}$ de colesterol/día, vía oral, a fin de inducir lesiones ateroescleróticas. Los conejos consumieron de manera voluntaria 9293,13 $\mathrm{ml}$ y $9930 \mathrm{ml}$ de leche con baja y alta relación AGt/AGS, respectivamente (p 0,404). Los conejos que consumieron leche con alta relación AGt/AGS registraron un leve incremento significativo (p 0,049) del colesterol total $(6,08 \mathrm{~g} / 1)$, cuando se comparó con el grupo control $(5,58 \mathrm{~g} / \mathrm{l})$. No se detectaron diferencias entre grupos en el colesterol LDL (p 0,073), colesterol HDL (p 0,078) y triglicéridos ( p 0,174). Las lesiones ateroescleróticas fueron más extensas en los conejos que consumieron leche con alta relación AGt/AGS, aunque sin significación estadística $(\mathrm{p}>0,05)$ cuando se comparó el control. En conclusión, la leche bubalina obtenida con la suplementación lipídica referida en este estudio, incrementó la colesterolemia y tendió a agravar la ateroesclerosis en conejos. Dichos efectos, posiblemente estén relacionados con el alto contenido de AGt de esta leche.
\end{abstract}

Palabras claves: búfalas, ácidos grasos trans, colesterol, ateroesclerosis.

\begin{abstract}
Lertora, W.J.; Villordo, G.I.; Mussart, N.B.; Catuogno, M.S.; S. Negrette, M.: Bubaline milk with a high content of trans fatty acids increased cholesterolemia and tended to aggravate atherosclerosis in rabbits. Rev. Vet. 30: 1, 32-38, 2019. Buffalo heifers supplemented with a mixture of sunflower oil + fish can produce milk with low level saturated fatty acids (SFA, which are considered hypercholesterolemic and pro-atherogenic), and increase the synthesis of trans-11 18: 1 and cis-9, trans-11 18: 2 fatty acids (with anti-atherogenic properties). This work compared the effects of the administration to rabbits of two bubaline milk, with low and high trans/saturated fatty acids $(t \mathrm{FA} / \mathrm{SFA})$ ratio, on the development of atherosclerosis induced by cholesterol. Twenty New Zealand rabbits, males, were randomly separated into a control group $(\mathrm{n}=10)$ that received milk with low $t \mathrm{FA} / \mathrm{SFA}$ ratio $(5.3 \% t \mathrm{FA}$ and $67.12 \%$ SFA) obtained from grazing buffalos; and a high trans group $(\mathrm{n}=10)$ that received milk with high $t$ FA/SFA ratio (25.84\% $t$ FA and $45.89 \%$ SFA) obtained from buffalos with lipid supplementation in their diet. The experience lasted 75 days and, from day 15, all rabbits were challenged with $0.93 \mathrm{~g}$ of cholesterol/day orally, to induce atherosclerotic lesions. The rabbits voluntarily consumed $9293.13 \mathrm{ml}$ and $9930 \mathrm{ml}$ of milk with low and high $t$ FA/SFA ratio, respectively (p 0.404). Rabbits that consumed milk with a high $t$ FA/SFA ratio increased ( $p$ 0.049) serum levels of total cholesterol (6.08 g/l), compared to the control group
\end{abstract}


$(5.58 \mathrm{~g} / \mathrm{l})$. No differences were detected between groups in LDL cholesterol (p 0.073), HDL cholesterol ( $p$ 0.078) and triglycerides ( $p$ 0.174). Atherosclerotic lesions were more extensive in rabbits that consumed milk with a high $t$ FA/SFA ratio, although without statistical significance ( $p>0.05$ ) compared to rabbits that consumed milk with low $t F A / S F A$ ratio. In conclusion, bubaline milk obtained from animals with lipid supplementation contributed to increase cholesterolemia and tended to worsen atherosclerosis in rabbits. These effects may be related to the high content of $t$ FA of this milk.

Key words: buffalos, trans fatty acids, cholesterol, atherosclerosis.

\section{INTRODUCCIÓN}

El relativamente alto contenido de ácidos grasos saturados (AGS) de la leche ha sido el principal motivo de crítica de los especialistas en nutrición hacia los productos lácteos, asumiendo que los alimentos ricos en grasas saturadas elevan la colesterolemia y contribuyen a la ateroesclerosis ${ }^{6}$. Este aspecto resulta importante ya que según el poder adquisitivo y los hábitos alimenticios de la población, los lácteos pueden aportar hasta el $60 \%$ del total de grasas saturadas que un ser humano consume diariamente ${ }^{4}$.

Consecuentemente, se han desarrollado estrategias nutricionales en el ganado lechero para reducir los niveles de AGS de cadena media, con efectos hipercolesterolémico y aterogénico, e incrementar la concentración de ácidos grasos insaturados (AGI) con efectos antimutagénico, hipocolesterolémico y ateroprotector en la leche ${ }^{14}$.

Disminuir los niveles de AGS e incrementar los AGI (particularmente cis-9 18:1; cis-9, trans-11 18:2; trans-11 18:1 y cis-9,cis-12,cis-15 18:3) en la leche y sus productos, ha mostrado tener efecto hipolipidémico en las personas ${ }^{11,15}$. Sin embargo, otros trabajos no reportaron efectos sobre la lipemia en personas ${ }^{3,16,18}$; mientras que otros hallaron un efecto perjudicial en el lipidograma sérico de conejos ${ }^{13}$ y seres humanos ${ }^{8}$, atribuidos a un sustancial incremento de AGt en la leche. Estas discrepancias se deben a diferencias en la metodología, características fisiopatológicas de los individuos $\mathrm{y}$, principalmente, a las diferentes dosis $\mathrm{y}$ composición de las grasas lácteas experimentales.

Estas modificaciones en el perfil de ácidos grasos de la leche fueron exploradas en búfalas mediante la suplementación de su dieta con aceite de girasol + pescado. Se consideró que dicha suplementacion mejoró la calidad nutricional de la grasa láctea bubalina; es decir, sería más apta para el consumo humano, ya que redujo los niveles de AGS e incrementaron los AGI, particularmente de trans-11 18:1 y cis-9, trans-11 18:2, en la leche bubalina ${ }^{9}$.

Teniendo en cuenta los antecedentes, este trabajo evaluó el efecto de dos leches bubalinas experimentales, con alta y baja relación AGt/AGS, en el desarrollo de ateroesclerosis en conejos hipercolesterolémicos.

Nuestra elección del modelo biológico experimental se fundamentó en que el conejo hipercolestero- lémico presenta un patrón lipoproteico similar al del ser humano hipercolesterolémico, con predominio de lipoproteínas de baja densidad (LDL) y una abundante actividad plasmática de la enzima de transferencia de esteres de colesterol, condición que naturalmente lo predispone a la ateroesclerosis ${ }^{19}$.

$\mathrm{Al}$ igual que en otras investigaciones, el conejo fue empleado para estudiar los efectos de la grasa láctea sobre la colesterolemia, perfil de lipoproteínas y lesiones sudanofílicas en aorta ${ }^{2,13}$.

\section{MATERIAL Y MÉTODOS}

Leche bubalina (Tabla 1): Dos leches experimentales con diferente perfil de ácidos grasos fueron obtenidas de búfalas con y sin suplementación lipídica. La leche control (sin suplementación lipídica) presentó baja relación AGt/AGS (5,3 y 67,12\%, respectivamente) y fue obtenida de búfalas alimentadas con pastura natural y $2 \mathrm{~kg}$ de maíz/día. La leche con alta relación $\mathrm{AG} t /$ AGS (25,84 y 45,89\%, respectivamente) fue obtenida de búfalas alimentadas con pastura natural y suplementadas diariamente con una mezcla de $210 \mathrm{ml}$ de aceite de girasol $+90 \mathrm{ml}$ de aceite de pescado vehiculizado en $2 \mathrm{~kg}$ de maíz durante 24 días. El contenido de colesterol (Laboratorio Físico-Químico, INTI, Buenos Aires) no difirió (prueba t bilateral para muestras independientes $=\mathrm{p} 0,727)$ entre la leche control $(10,30 \pm 2,27 \mathrm{mg} / 100 \mathrm{~g})$ y la leche alta relación AGt/AGS $(9,68 \pm 2,55 \mathrm{mg} / 100 \mathrm{~g})$.

Animales, administración de leche y diseño experimental: Veinte conejos machos Neozelandeses (suministrados por el Centro de Medicina Comparada, FCV-UNL, Esperanza, Santa Fe), con un peso corporal de 2,02 $\pm 0,25 \mathrm{~kg}$, fueron divididos aleatoriamente en dos grupos de 10 animales, alojados en jaulas metálicas individuales, en una habitación con temperatura controlada $\left(22^{\circ} \mathrm{C}\right)$, con ciclo de luz/oscuridad de 12 horas y con acceso al agua de bebida y al alimento balanceado ad libitum. Los conejos recibieron leche control (grupo control) y leche alta relación AGt/AGS (grupo alto trans) durante 75 días. La leche fue administrada en forma fluida y su consumo fue voluntario; restringiendo su consumo diario a un máximo de $200 \mathrm{ml} /$ conejo hasta el día 45 y a un máximo de $100 \mathrm{ml} /$ conejo hasta el final de la experiencia. Durante los primeros 15 días de la experiencia se determinaron los efectos del consumo de las leches bubalinas sobre el lipidograma sérico en 
conejos saludables (sin administración de colesterol). Durante los 60 días posteriores, se indujo hipercolesterolemia mediante la administración de colesterol vía oral $(0,93 \mathrm{~g}$ de colesterol $94 \% /$ conejo/día) y se determinaron los efectos del consumo de las leches sobre el lipidograma sérico y el desarrollo de ateroesclerosis en aorta en conejos con hipercolesterolemia. Se registró diariamente el consumo de leche y de alimento balanceado por animal. El peso corporal se verificó semanalmente. Todos los procedimientos de este estudio fueron aprobados por el Comité de Ética y Bioseguridad, FCV-UNNE, protocolo $\mathrm{N}^{\circ} 017$.

Extracción de sangre y lipidograma sérico: La extracción de sangre se realizó los días $0,15,45$ y 75 en todos los conejos, con ayuno de 8 horas. Se determinaron triglicéridos (técnica del glicerolfosfato-oxidasa/ peroxidasa, $505 \mathrm{~nm}$ ), colesterol total (colesterol-oxidasa-peroxidasa, $505 \mathrm{~nm}$ ), colesterol ligado a lipoproteínas de alta densidad (C-HDL) y de baja densidad (CLDL): por precipitación selectiva de la lipoproteína y valoración enzimática de colesterol (reactivos Wiener).

Necropsia y toma de muestras: Cumplido los 75 días de la experiencia, se procedió a la eutanasia y necropsia de los animales. Se disecó la aorta completa, que fue fijada en formol bufferado al $10 \%$ durante 24 horas.

Cuantificación de lesiones ateroescleróticas en aorta: Luego de la fijación, las aortas completas fueron coloreadas con Sudan IV para evidenciar depósitos lipídicos en el estrato intimal. La superficie endotelial fue fotografiada y las imágenes digitales procesadas con el software ImageJ versión 1.47 n (U.S. National Institutes of Health, Bethesda, Maryland, USA) para medir el área endotelial total y el área sudanofílica. Las lesiones ateroescleróticas en cada aorta fueron expresadas en porcentaje de área sudanofílica que ocupaba la superficie endotelial total.

Histomorfometría de aorta: Un segmento de aorta ascendente ( $5 \mathrm{~mm}$ antes del tronco braquiocefálico) fue procesado según la técnica histológica clásica para bloques parafinados. Se realizaron cortes seriados a $5 \mu \mathrm{m}$ que fueron coloreados con hematoxilina y eosina (HE) y procesados con una técnica inmunohistoquímica (IHQ) para la detección de macrófagos (clon RAM11, dilución 1:50; Dako) y de músculo liso (clon HHF35, dilución 1:100; Dako) como previamente fue descripto ${ }^{20}$. En las secciones teñidas con HE se midió en micrómetros, con objetivo de 10x, el espesor intimal (desde la superficie luminal hasta la membrana elástica interna) en el sitio de máximo espesor. Las lesiones en aorta fueron clasificadas en estría grasa (intima infiltrada con macrófagos espumosos), placa en transición (íntima infiltrada con macrófagos espumosos, músculo liso y depósito de matriz extracelular) y ateroma (íntima con capa fibrosa de músculo liso y matriz extracelular cubriendo un centro de macrófagos espumosos con focos de necrosis y cristales de colesterol extracelular). Las secciones inmunoteñidas fueron fotografiadas y las imágenes fueron procesadas (software ImageJ) para
Tabla 1. Composición de ácidos grasos en leche de búfalas sin suplementación lipídica (leche control) y suplementadas con $300 \mathrm{ml} /$ día de una mezcla (70:30 $\mathrm{wt} / \mathrm{wt}$ ) de aceite de girasol y de pescado durante 24 días (leche alta relación AGt/AGS).

\begin{tabular}{|c|c|c|}
\hline $\begin{array}{l}\text { ácidos grasos } \\
\text { ( } \mathrm{g} / 100 \mathrm{~g} \text { de grasa) }\end{array}$ & leche control & $\begin{array}{c}\text { leche alta relación } \\
\text { AGt/AGS }\end{array}$ \\
\hline $4: 0$ & $3,86 \pm 0,38$ & $1,86 \pm 0,90^{*}$ \\
\hline $6: 0$ & $1 \pm 0$ & $0,46 \pm 0,11 *$ \\
\hline $8: 0$ & $0,51 \pm 0,09$ & $0,14 \pm 0,05^{*}$ \\
\hline 10:0 & $0,99 \pm 0,04$ & $0,33 \pm 0,05^{*}$ \\
\hline $10: 1$ & $0,03 \pm 0,01$ & $0,01 \pm 0^{*}$ \\
\hline $11: 0$ & $0,01 \pm 0$ & $0,01 \pm 0$ \\
\hline $12: 0$ & $1,29 \pm 0,49$ & $1 \pm 0$ \\
\hline $12: 1$ & $0,09 \pm 0,02$ & $0,10 \pm 0$ \\
\hline $14: 0$ & $8,14 \pm 1,07$ & $4 \pm 0,82^{*}$ \\
\hline $14: 1$ & $0,73 \pm 0,14$ & $0,54 \pm 0,05^{*}$ \\
\hline $15: 0$ iso & $0,30 \pm 0,08$ & $0,09 \pm 0,02 *$ \\
\hline $15: 0$ & $1,43 \pm 0,53$ & $1 \pm 0$ \\
\hline $15: 1$ & $0,36 \pm 0,08$ & $0,21 \pm 0,04^{*}$ \\
\hline $16: 0$ & $27,57 \pm 2,70$ & $20,57 \pm 0,53 *$ \\
\hline $16: 1$ & $1 \pm 0$ & $1 \pm 0$ \\
\hline $17: 0$ & $1,14 \pm 0,38$ & $1 \pm 0$ \\
\hline $17: 1$ & $0,27 \pm 0,08$ & $0,17 \pm 0,05^{*}$ \\
\hline 18:0 & $21 \pm 2,45$ & $15 \pm 2,77^{*}$ \\
\hline trans-8 18:1 & $0,49 \pm 0,07$ & $1,71 \pm 0,49^{*}$ \\
\hline trans-9 18:1 & $0,26 \pm 0,05$ & $1 \pm 0^{*}$ \\
\hline trans-10 18:1 & $0,26 \pm 0,08$ & $0,96 \pm 0,08^{*}$ \\
\hline trans-11 18:1 & $4,29 \pm 1,25$ & $22,14 \pm 3,44^{*}$ \\
\hline cis-9 18:1 & $21,29 \pm 1,50$ & $19,14 \pm 2,73$ \\
\hline cis-11 18:1 & $0,67 \pm 0,05$ & $1,86 \pm 0,38^{*}$ \\
\hline trans-9,trans-12 18:2 & $0,01 \pm 0$ & $0,03 \pm 0,01^{*}$ \\
\hline cis-9,cis-12 18:2 & $0,97 \pm 0,05$ & $1 \pm 0$ \\
\hline cis-9,trans-11 18:2 & $1 \pm 0$ & $3,57 \pm 0,79 *$ \\
\hline cis-9,cis-12,cis-15 18:3 & $0,54 \pm 0,14$ & $0,27 \pm 0,05^{*}$ \\
\hline 20:4 (ARA) & $0,09 \pm 0,01$ & $0,05 \pm 0,02 *$ \\
\hline 20:5 (EPA) & $0,06 \pm 0,01$ & $0,05 \pm 0,01$ \\
\hline 22:6 (DHA) & $0,01 \pm 0$ & $0,01 \pm 0$ \\
\hline$\Sigma$ AGS & $67,12 \pm 2,07$ & $45,89 \pm 2,37^{*}$ \\
\hline$\Sigma 12+14+16$ & $37 \pm 3,87$ & $25,57 \pm 0,98^{*}$ \\
\hline$\Sigma$ cis AGM & $24,44 \pm 1,65$ & $23,03 \pm 2,43$ \\
\hline$\Sigma$ cis AGP & $1,67 \pm 0,17$ & $1,39 \pm 0,07^{*}$ \\
\hline$\sum$ trans\# & $5,30+1,26$ & $25,84 \pm 3,76^{*}$ \\
\hline
\end{tabular}

*p valor $\leq 0,05$ (prueba t bilateral para muestras independientes). ARA: ácido araquidonico. EPA: ácido eicosapentaenoico. DHA: ácido docosahexaenoico. AGS: ácidos grasos saturados. AGM: ácidos grasos monoinsaturados. AGP: ácidos grasos poliinsaturados. \# La suma no incluye a cis-9, trans-11 18:2.

medir el área de lesión intimal en $\mathrm{mm}^{2}$ y el porcentaje de macrófagos y músculo liso de dichas áreas.

Análisis estadístico: Los resultados fueron expresados en media \pm desvío estándar $(\overline{\mathrm{X}} \pm \mathrm{DE})$. La comparación de las medias de la ganancia de peso, consumo de alimento, consumo de leche, área sudanofílica, espesor intimal, composición de macrófagos y músculo liso de las lesiones, fueron obtenidos con la prueba $\mathrm{T}$ 
Tabla 2. Consumo de leche, alimento balanceado y peso corporal, según grupos.

\begin{tabular}{lcc}
\hline & grupo control & grupo alto trans \\
\hline consumo de leche $(\mathrm{ml})$ & $9293,13 \pm 1770,74^{\mathrm{a}}$ & $9930,00 \pm 1557,88^{\mathrm{a}}$ \\
consumo diario de alimento $(\mathrm{g})$ & $77,19 \pm 26,74^{\mathrm{a}}$ & $69,25 \pm 34,80^{\mathrm{a}}$ \\
peso corporal inicial $(\mathrm{kg})$ & $2,05 \pm 0,23^{\mathrm{a}}$ & $2,00 \pm 0,27^{\mathrm{a}}$ \\
peso corporal final $(\mathrm{kg})$ & $3,13 \pm 0,32^{\mathrm{a}}$ & $3,10 \pm 0,40^{\mathrm{a}}$ \\
ganancia de peso $(\mathrm{kg})$ & $1,08 \pm 0,27^{\mathrm{a}}$ & $1,10 \pm 0,32^{\mathrm{a}}$ \\
\hline
\end{tabular}

${ }^{a}$ En cada columna, medias con una letra común no son significativamente diferentes (prueba $t$ para muestras independientes, $\mathrm{p}>0,05$ ).

Tabla 3. Evolución del lipidograma sérico según grupo.

\begin{tabular}{|c|c|c|c|c|}
\hline tasa sérica $(\mathrm{g} / \mathrm{l})$ & tasa basal & día 15 & día 45 & día 75 \\
\hline \multicolumn{5}{|l|}{ C-total } \\
\hline control & $0,71 \pm 0,21$ & $1,01 \pm 0,18^{\mathrm{a}}$ & $8,44 \pm 0,77^{b c}$ & $7,28 \pm 1,65^{\mathrm{d}}$ \\
\hline alto trans & $0,70 \pm 0,21$ & $1,00 \pm 0,14^{\mathrm{a}}$ & $9,01 \pm 0,55^{\mathrm{b}}$ & $8,22 \pm 0,08^{\mathrm{cd}}$ \\
\hline \multicolumn{5}{|l|}{ C-HDL } \\
\hline control & $0,41 \pm 0,08$ & $0,56 \pm 0,10^{\mathrm{a}}$ & $2,97 \pm 0,82^{b c}$ & $2,17 \pm 1,04^{\mathrm{d}}$ \\
\hline alto trans & $0,36 \pm 0,09$ & $0,49 \pm 0,12^{\mathrm{a}}$ & $3,45 \pm 0,11^{\mathrm{b}}$ & $2,44 \pm 0,66^{\mathrm{cd}}$ \\
\hline \multicolumn{5}{|l|}{ C-LDL } \\
\hline control & $0,19 \pm 0,11$ & $0,23 \pm 0,07^{\mathrm{a}}$ & $5,86 \pm 1,00^{b c}$ & $4,83 \pm 1,59^{d}$ \\
\hline alto trans & $0,14 \pm 0,09$ & $0,25 \pm 0,11^{\mathrm{a}}$ & $6,32 \pm 0,27^{\mathrm{b}}$ & $5,75 \pm 0,10^{\mathrm{cd}}$ \\
\hline \multicolumn{5}{|l|}{ triglicéridos } \\
\hline control & $0,48 \pm 0,13$ & $0,49 \pm 0,16^{\mathrm{a}}$ & $0,91 \pm 0,55^{\mathrm{a} b}$ & $2,47 \pm 2,57^{b c}$ \\
\hline alto trans & $0,57 \pm 0,21$ & $0,42 \pm 0,14^{\mathrm{a}}$ & $2,37 \pm 2,13^{b c}$ & $3,12 \pm 2,43^{\mathrm{c}}$ \\
\hline
\end{tabular}

para muestras independientes. Se realizó un análisis de modelos lineales mixtos para comparar las medias y estudiar el efecto del tiempo de las variables del lipidograma sérico (colesterol total, C-HDL, C-LDL y triglicéridos). El modelo con una correlación residual sin estructura, varianzas residuales heterogéneas en el tiempo y sin efecto aleatorio, fue el seleccionado para describir los datos del lipidograma sérico. Las diferencias entre los grupos fueron declaradas significativas con $\mathrm{p} \leq 0,05$.

\section{RESULTADOS}

Consumo de leche, de alimento balanceado y peso corporal (Tabla 2): todos los conejos aceptaron de forma voluntaria ambos tipos de leche. A lo largo de la experiencia, cada conejo consumió 9293,13 $\pm 1770,74$ $\mathrm{ml}$ de leche bubalina control y $9930 \pm 1557,88 \mathrm{ml}$ de leche con alta relación AGt/AGS, sin diferencias entre grupos ( $p$ 0,404). Tampoco se observaron diferencias entre los grupos en el consumo de leche entre los días 1 y 14 (p 0,385$)$, días 15 y $44(\mathrm{p} 0,181)$ y días 45 y 75 (p 0,909$)$ de la experiencia (datos no mostrados en Tabla 2). El registro del consumo de alimento balanceado no detectó diferencias entre los grupos ( $\mathrm{p} 0,486$ ). Los conejos de ambos grupos ganaron peso a lo largo de la experiencia, sin diferencias en peso corporal inicial ( $p$ $0,683)$, peso corporal final $(\mathrm{p} 0,860)$ y ganancia de peso (p 0,892).

Lipidograma sérico: Los conejos que consumieron leche con alta relación AGt/AGS registraron un leve incremento significativo (p 0,049) del colesterol total $(6,08 \mathrm{~g} / \mathrm{l})$, cuando se comparó con el grupo control (5,58 g/l). El colesterol LDL fue más elevado en el grupo que recibió leche con alta relación AGt/AGS (4,10 g/l) que el grupo control $(3,64 \mathrm{~g} / 1)$, pero el incremento no fue significativo (p 0,073$)$. No se detectaron diferencias en los registros del colesterol HDL entre los grupos alto trans $(2,13 \mathrm{~g} / \mathrm{l})$ y control $(1,9 \mathrm{~g} / \mathrm{l})$ (p 0,078). Tampoco se detectaron diferencias $(\mathrm{p} 0,174)$ en la trigliceridemia de los grupos alto trans $(1,97 \mathrm{~g} / \mathrm{l})$ y control $(1,29 \mathrm{~g} / \mathrm{l})$. Cuando se evaluó la colesterolemia en el tiempo (Tabla 3), las tasas séricas basales de colesterol total, colesterol HDL y colesterol LDL se incrementaron en ambos grupos, sin diferencias significativas. Entre los días 45 y 75 se registró un leve descenso de la colesterolemia en ambos grupos, datos previstos por la restricción en el consumo de leche durante este período.

Ateroesclerosis en aorta (Tabla 4): todos los conejos en ambos grupos desarrollaron lesiones sudanofílicas en aorta (Figura 1). Los conejos que recibieron leche con alta relación AGt/AGS desarrollaron mayor extensión del área sudanofílica, del espesor intimal y del área de lesión intimal en aorta; sin embargo, las diferencias no fueron significativos ( $\mathrm{p} 0,5776, \mathrm{p} 0,2014$ y p 0,283 , respectivamente). En la evaluación microscópica, todos los conejos de ambos grupos desarrollaron lesiones ateroescleróticas del tipo placas en transición

Tabla 4. Efectos de leches bubalinas con baja (control) y alta relación AGt/AGS (grupo alto trans) en el desarrollo de ateroesclerosis en aorta de conejos hipercolesterolémicos.

\begin{tabular}{lccccc}
\hline grupo & $\begin{array}{c}\text { área sudano-fílica } \\
(\%)\end{array}$ & $\begin{array}{c}\text { espesor intimal } \\
(\mu \mathrm{m})\end{array}$ & $\begin{array}{c}\text { área de lesión } \\
\text { intimal }\left(\mathrm{mm}^{2}\right)\end{array}$ & $\begin{array}{c}\text { área de macrófagos } \\
(\%)\end{array}$ & $\begin{array}{c}\text { área de músculo liso } \\
(\%)\end{array}$ \\
\hline control & $21,48 \pm 16,54^{\mathrm{a}}$ & $261,51 \pm 162,84^{\mathrm{a}}$ & $0,757 \pm 0,40^{\mathrm{a}}$ & $21,90 \pm 13,40^{\mathrm{a}}$ & $45,65 \pm 12,90^{\mathrm{a}}$ \\
alto trans & $26,38 \pm 17,88^{\mathrm{a}}$ & $305,82 \pm 176,19^{\mathrm{a}}$ & $1,343 \pm 0,96^{\mathrm{a}}$ & $21,67 \pm 11,98^{\mathrm{a}}$ & $45,92 \pm 16,79^{\mathrm{a}}$ \\
\hline
\end{tabular}

a: En cada columna, medias con una letra común no son significativamente diferentes (prueba $t$ para muestras independientes, $\mathrm{p}>0,05)$. 


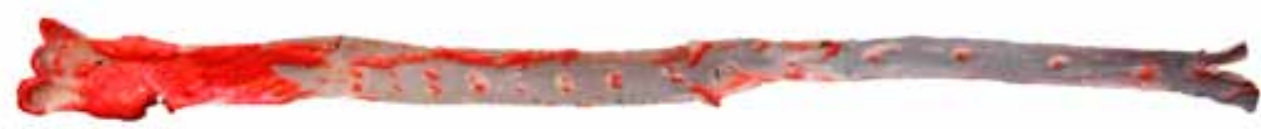

alto AGt

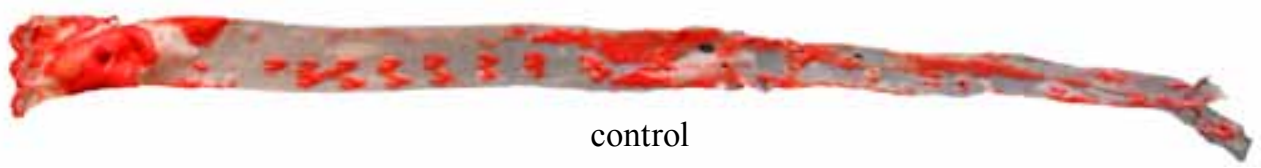

Figura 1. Lesiones sudanofílicas en aortas de conejos hipercolesterolémicos que consumieron leche bubalina con baja (control) y alta relación AGt/AGS (alto AGt).

en la aorta ascendente (Figura 2). En dichas lesiones, el porcentaje del área ocupada por macrófagos y células musculares lisas no difirió entre los grupos ( $\mathrm{p}$ 0,945 y p 0,840 , respectivamente).

\section{DISCUSIÓN}

La reducción de los AGS y el incremento de los AGI en la leche lograron mejorar el lipidograma sérico de personas ${ }^{7,10}$ y ratas ${ }^{1}$. Así, personas normolipidémicas que consumieron grasa láctea reducida en AGS (63,3\%), con un incremento moderado de AGt (4\%), cis-AGMI (25,6\%), cis-AGPI (2,87\%) y cis-9,trans-11 18:2 (0,67\%) durante 21 días, redujeron su colesterol total y colesterol-LDL, comparadas con el grupo de personas normolipidémicas que consumieron grasa láctea comercial (72\% de AGS, 2,9\% de AGt, 19,5\% de cis-AGMI, 2,21 $\%$ de $c i s$-AGPI y $0,42 \%$ de cis-9,trans-11 18:2) ${ }^{7}$.

Similar respuesta del lipidograma sérico fue registrada en personas con moderada hipercolesterolemia al suplementar su dieta durante 21 días con 90 g/día de queso de oveja naturalmente enriquecido con cis9,cis-12,cis-15 18:3 (2,1\%), cis-9,trans-11 18:2 (2,8\%) y trans-11 18:1 (6,3\%) y bajo contenido de AGS (46\%), cuando se comparó con un queso control (cis-9,cis12,cis-15 18:3: 0,6\%, cis-9,trans-11 18:2: 1\%, trans-11 18:1: 1,7\% y AGS: 59\%) ${ }^{10}$.

Por el contrario, nuestros resultados demostraron que una leche reducida en AGS $(45,8 \%)$ y con un sustancial incremento de AGt $(25,8 \%)$ indujo un leve pero significativo incremento del colesterol total plasmático en conejos, al compararla con el grupo de conejos que recibió leche control (67\% de AGS y 5,3\% de AGt).

Nuestros resultados concuerdan con los reportados en seres humanos y cobayos que recibieron lácteos con alta relación AGt/AGS. En humanos, los AGt de origen rumiante, cuyo principal isómero es trans-11 18:1, tuvieron un efecto neutro en los lípidos plasmáticos cuando la ingesta fue baja $(0,8 \%$ de calorías/día aportado por AGt) o moderada ( $1,5 \%$ de la ingesta calórica diaria aportada por AGt); mientras que causó hipercolesterolemia e incrementó las subfracciones aterogénicas de las lipoproteínas cuando su ingesta fue alta $(3,3 \%$ o $3,7 \%$ de la ingesta calórica diaria aportada por AGt $)^{5,8}$.

En cobayos hipercolesterolémicos, dos dietas con altas dosis de AGt industriales y AGt rumiante $(\sim 15 \mathrm{~g}$
AGt $/ 100 \mathrm{~g}$ de lípidos, en ambos tipos de dietas) tuvieron similar efecto sobre la lipemia (incrementos del colesterol total, C-LDL, C-HDL y triglicéridos), a pesar de su distribución isomérica diferente (trans-11 18:1 comprendió el 76,5\% del total de isómeros trans 18:1 en la grasa rumiante; mientras trans $-6-8$, trans -9 y trans -10 18:1 fueron los más abundantes en la grasa vegetal hidrogenada) ${ }^{12}$.

El grupo que consumió leche con alta relación AGt/ AGS registró mayor extensión de lesiones ateroescleróticas en aorta y, aunque estas variables no alcanzaron el nivel de significación estadística, están en concordancia con el incremento del colesterol total registrado en este grupo de conejos. La falta de efecto ateroprotector de la leche con alta relación AGt/AGS de la presente experiencia, concuerda con los efectos perjudiciales de la grasa láctea con altos niveles de AGt $(11,8 \%$ de trans-10 C18:1 y 1,8\% de trans-11 18:1) y reducida en AGS (41,9\%) reportado previamente en conejos ${ }^{2,13}$.

A diferencia de nuestros resultados, otros han demostrado que la grasa láctea con bajos niveles de AGS y altas concentraciones de AGI son menos aterogénicas. Hámsteres hiperlipidémicos alimentados con grasa láctea reducida en $\mathrm{AGS}(66,77 \%)$ y enriquecidas con trans-11 18:1 (4,85\%), cis-9,trans-11 18:2 (2,38\%) y cis9,cis-12,cis-15 18:3 (1,22\%) (leche bovina de sistema pastoril) presentaron $25 \%$ menos depósito de colesterol en aorta con relación a los hámsteres alimentados con grasa láctea comercial (de bovinos alimentados con henificados y ensilados), con mayor concentración de AGS (72,05\%) y bajos niveles de AGI $(0,97 \%$ de trans-11 18:1 0,37\% de cis-9,trans-11 18:2 y 0,3\% de cis-9,cis-12,cis-15 18:3).

Dicho efecto ateroprotector fue atribuido al mejor perfil de lipoproteínas séricas (bajo niveles de C-LDL, altos niveles de C-HDL y baja relación C-LDL / CHDL) y a la menor reacción inflamatoria de la aorta (menor expresión de VCAM-1, IL-1 y COX-2 en aorta) inducidos por la mayor concentración de AGI ${ }^{17}$.

Nuestros resultados tienden a sostener el concepto que un sustancial incremento de AGt en la leche puede inducir incrementos en la colesterolemia y contrarrestar el efecto ateroprotector que se procura con la reducción de los AGS e incremento del cis-9,trans-11 18:2. No obstante, no puede descartarse que la ligera reducción de cis-9,cis-12,cis-15 18:3 también pudo con- 
tribuir a la ausencia de efecto ateroprotector de la leche bubalina con alta relación AGt/AGS.

En conclusión, la leche bubalina obtenida con la suplementación lipídica referida en este estudio, perjudi-
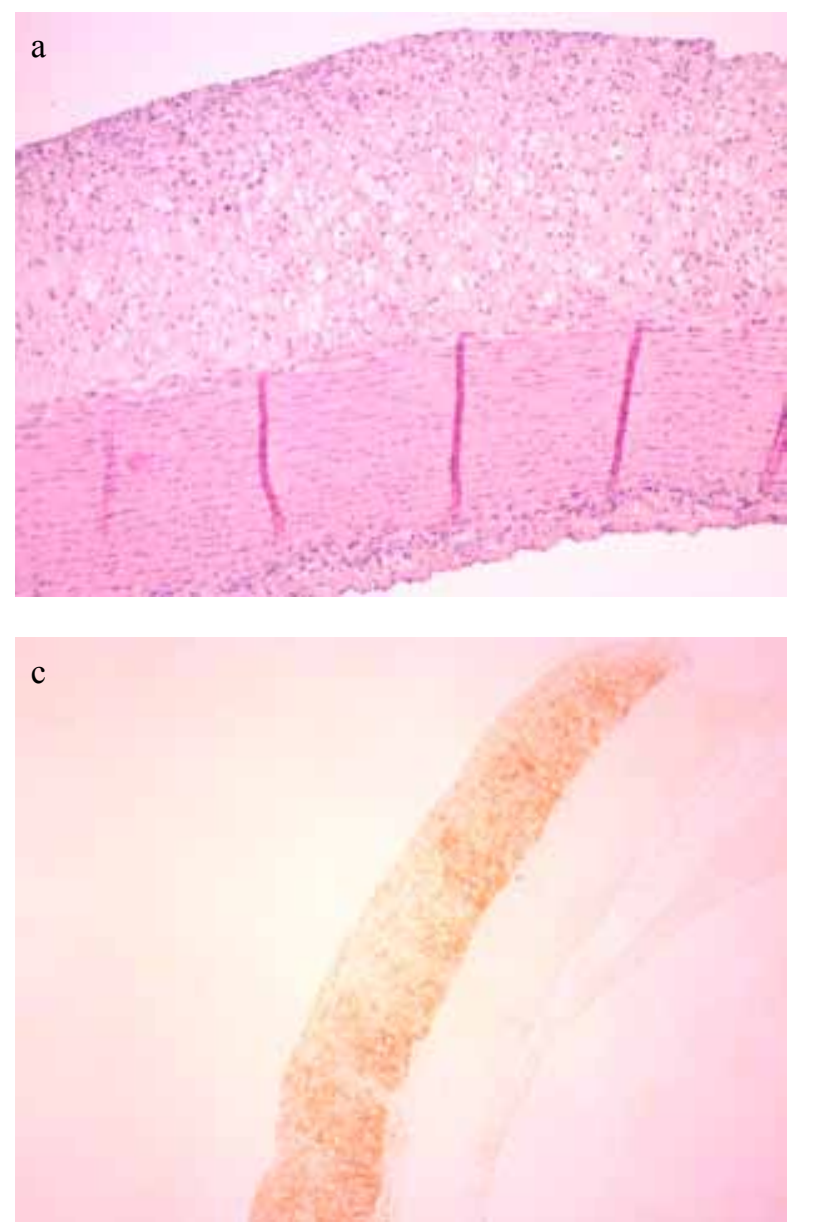

e

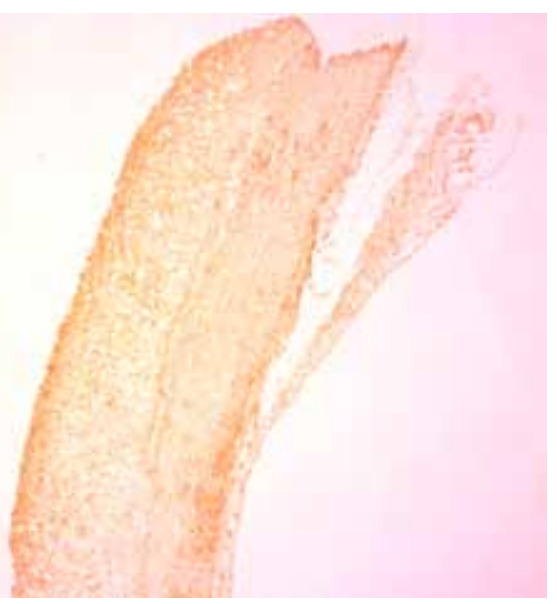

có la colesterolemia y tendió a incrementar las lesiones ateroescleróticas en conejos. Dichos efectos estuvieron posiblemente relacionados con el alto contenido de AGt de la leche de búfalas.
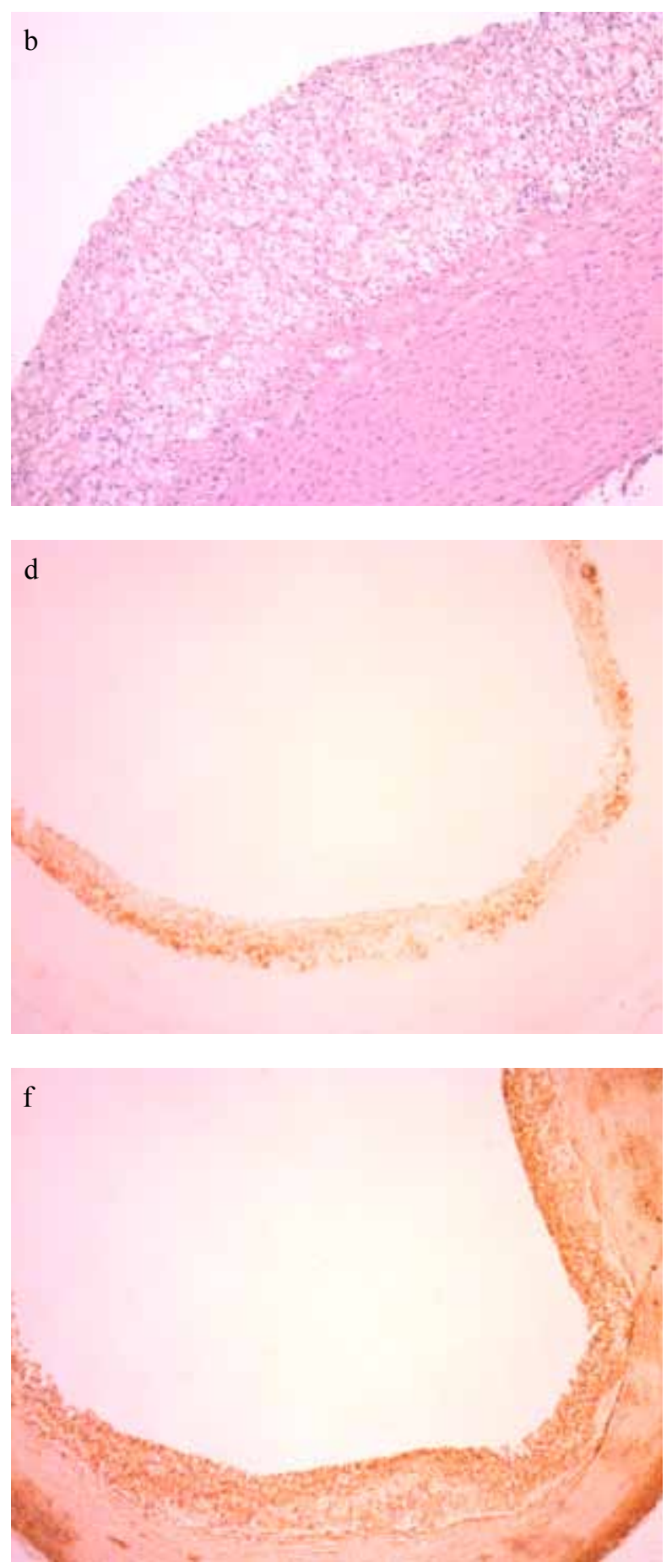

Figura 2. Efectos del consumo de leches bubalinas con baja relación AGt/AGS (a, c y e) y con alta relación AGt/AGS (b, d y f) en el desarrollo de ateroesclerosis en conejos hipercolesterolémicos. a: Aorta ascendente de conejo control con engrosamiento del estrato intimal debido a infiltración de macrófagos espumosos y células musculares lisas (placa en transición). b: El consumo de leche con alta relación AGt/AGS no modificó el tipo de lesión ateroesclerótica, clasificada como placa en transición (H-E, obj. 10x). c: Inmunomarcación de macrófagos en lesión intimal de aorta ascendente de conejo control. d: La leche con alta relación AGt/AGS no modificó la infiltración intimal de macrófagos (anticuerpo RAM11, obj. 5x). e: Inmunomarcación de células musculares lisas en lesión intimal de aorta ascendente de conejo control. f: La leche con alta relación AGt/AGS no modificó la infiltración intimal de células musculares lisas (anticuerpo HHF35, obj. 5x). 


\section{REFERENCIAS}

1. Anadón A et al. 2010. Acute oral safety of dairy fat rich in trans-10 C18:1 versus vaccenic plus conjugated linoleic acid in rats. Food Chem Tox 48: 591-598.

2. Bauchart D et al. 2007. Butters varying in trans 18:1 and cis-9,trans-11 conjugated linoleic acid modify plasma lipoproteins in the hypercholesterolemic rabbit. Lipids 42 : 123-133.

3. Brown AW, Trenkle AH, Beitz DC. 2011. Diets high in conjugated linoleic acid from pasture-fed cattle did not alter markers of health in young women. Nutr Res 31: 33-41.

4. Gagliostro GA. 2007. Producción de lácteos con alto impacto sobre la salud humana. Tecnología láctea latinoamericana, 45:56-63.

5. Gebauer SK, Destaillats F, Dionisi F, Krauss RM, Baer DJ. 2015. Vaccenic acid and trans fatty acid isomers from partially hydrogenated oil both adversely affect LDL cholesterol: double-blind, randomized controlled trial. Am J Clin Nutr 102: 1339-1346.

6. Haug A, Hostmark AT, Harstad OM. 2007. Bovine milk in human nutrition: a review. Lipids Health Dis 6: 25.

7. Malpuech BC et al. 2010. Differential impact of milk fatty acid profiles on cardiovascular risk biomarkers in healthy men and women. Eur J Clin Nutr 64: 752-759.

8. Motard BA et al. 2008. Study of the effect of trans fatty acids from ruminants on blood lipids and other risk factors for cardiovascular diseases. Am J Clin Nutr 87: 593-599.

9. Patiño EM et al. 2017. Perfil de ácidos grasos en leche de búfalas alimentadas con pastura natural y suplementadas con aceites de girasol y pescado. Rev Vet 28: 19-26.

10. Pintus $\mathbf{S}$ et al. 2013. Sheep cheese naturally enriched in $\alpha$-linolenic, conjugated linoleic and vaccenic acids improves the lipid profile and reduces anandamide in the plasma of hypercholesterolaemic subjects. Br J Nutr 109: 1453-1462.

11. Poppitt SD et al. 2002. Lipid-lowering effects of a modified butter-fat: a controlled intervention trial in healthy man. Eur J Clin Nutr 56: 64-71.
12. Rice BH, Kraft J, Destaillats F, Bauman DE, Lock AL. 2010. Ruminant-produced trans-fatty acids raise plasma total and small HDL particles concentrations in male Hartley guinea pigs. J Nutr 140: 2173-2179.

13. Roy A et al. 2007. Butters rich either in trans-10-C18:1 or in trans-11-C18:1 plus cis-9, trans-11 CLA differentially affect plasma lipids and aortic fatty streak in experimental atherosclerosis in rabbits. Animal 1: 467-476.

14. Shingfield KJ, Chilliard Y, Toivonen V, Kairenius P, Givens DI. 2008. Trans fatty acids and bioactive lipids in ruminant milk. Adv Exp Med Biol 606: 3-65.

15. Tholstrup T et al. 2006. Effects of butter high in ruminant trans and monounsaturated fatty acids on lipoproteins, incorporation of fatty acids into lipid classes, plasma C-reactive protein, oxidative stress, hemostatic variables, and insulin in healthy young men. Am J Clin Nutr 83: 237-243.

16. Tricon $\mathbf{S}$ et al. 2006. Effects of dietary products naturally enriched with cis-9,trans-11 conjugated linoleic acid on the blood lipid profile in healthy middle-aged men. Am J Clin Nutr 83: 744-753.

17. Valeille $\mathbf{K}$ et al. 2006. The natural concentration of conjugated linoleic acid, cis-9, trans-11, in milk fat has antiatherogenic effects in hyperlipidemic hamsters. $J$ Nutr 136: 1305-1310.

18. Venkatramanan S et al. 2010. Milk enriched with conjugated linoleic acid fails to alter blood lipids or body composition in moderately overweight, borderline hyperlipidemic individuals. J Am Coll Nutr 29: 152-159.

19. Yin W et al. 2012. Plasma lipid profiling across species for the identification of optimal animal model of human dyslipidemia. J Lipid Res 53: 51-65.

20. Zhang C et al. 2010. A practical method for quantifying atherosclerotic lesions in rabbits. J Comp Path 142: 122128. 